\title{
ESSENTIAL SPACEFLIGHT DYNAMICS \\ AND MAGNETOSPHERICS
}




\section{THE SPACE TECHNOLOGY LIBRARY}

Published jointly by Microcosm Press and Kluwer Academic Publishers

An Introduction to Mission Design for Geostationary Satellites, J.J. Pocha

Space Mission Analysis and Design, 1st edition, James R. Wertz and Wiley J. Larson Space Mission Analysis and Design, 2nd edition, Wiley J. Larson and James R. Wertz Space Mission Analysis and Design, 3rd edition, James R. Wertz and Wiley J. Larson Space Mission Analysis and Design Workbook, Wiley J. Larson and James R. Wertz Handbook of Geostationary Orbits, E.M. Soop

Spacecraft Structures and Mechanisms, From Concept to Launch, Thomas P. Sarafin

Spaceflight Life Support and Biospherics, Peter Eckart

Reducing Space Mission Cost, James R. Wertz and Wiley J. Larson

The Logic of Microspace, Rick Fleeter

Space Marketing: A European Perspective, Walter A.R. Peeters

Fundamentals of Astrodynamics and Applications, David A. Vallado

Influence of Phychological Factors on Product Development, Eginaldo S. Kamata

\section{The Space Technology Library Editorial Board}

Managing Editor: James R. Wertz, Microcosm, Inc., El Segundo, CA

Editorial Board: Val A. Chobotov, The Aerospace Corporation (retired)

Michael L. DeLorenzo, United States Air Force Academy

Roland Doré, International Space University, Strasbourg, France

Robert B. Giffen, United States Air Force Academy (retired)

Gwynne Gurevich, Microcosm, Inc.

Wiley J. Larson, United States Air Force Academy

Tom Logsdon, Rockwell International (retired)

Landis Markley, Goddard Space Flight Center

Robert G. Melton, Pennsylvania State University

Keiken Ninomiya, Institute of Space \& Astronautical Science, Japan

Jehangir J. Pocha, Matra Marconi Space, Stevenage, England

Frank J. Redd, Utah State University

Malcolm D. Shuster, University of Florida

Gael Squibb, Jet Propulsion Laboratory

Martin Sweeting, University of Surrey, England 


\section{Essential Spaceflight Dynamics and Magnetospherics}

\section{Boris V. Rauschenbakh}

Department of Theoretical Mechanics, Moscow Institute of Physics and Technology, Moscow, Russia

\section{Michael Yu. Ovchinnikov}

Keldysh Institute of Applied Mathematics, Russian Academy of Sciences, Moscow, Russia, and Department of Theoretical Mechanics, Moscow Institute of Physics and Technology, Moscow, Russia

\section{Susan McKenna-Lawlor}

Space Technology Ireland Ltd, National University of Ireland, Maynooth, Co. Kildare, Ireland 
eBook ISBN: $\quad 0-306-48027-1$

Print ISBN: $\quad$ 1-4020-1063-X

(C2004 Kluwer Academic Publishers

New York, Boston, Dordrecht, London, Moscow

Print C2003 Kluwer Academic Publishers

Dordrecht

All rights reserved

No part of this eBook may be reproduced or transmitted in any form or by any means, electronic, mechanical, recording, or otherwise, without written consent from the Publisher

Created in the United States of America

Visit Kluwer Online at:

http://kluweronline.com

and Kluwer's eBookstore at:

http://ebooks.kluweronline.com 
This book is dedicated to our esteemed parents

Victor Yakovlevich Rauschenbakh and Leontina Fedorovna Rauschenbakh

Yuriy Fedorovich Ovchinnikov and Antonina Spiridonovna Ovchinnikova

James Christopher McKenna and Margaret McKenna, née Keane

with deep appreciation of their endless love, guidance and inspiration. 


\section{Preface}

The present book Essential Spaceflight Dynamics and Magnetospherics describes, in the first instance, some of the key aspects of celestial mechanics and spaceflight dynamics. We begin with classical two and three body problems illustrative of the aesthetic aspects of applying analytical methods of investigation to celestial mechanics. Then, osculating orbital elements are introduced as well as analysis techniques sufficient to evaluate the influence of various disturbing forces on spacecraft. Next a theory of manoeuvres is outlined and the methodology of making interplanetary trajectory corrections. Ideas involving various approaches to orbital element determinations using measured data are also considered.

The forces applied to a spacecraft can result in the development of torques that influence attitude motion and the effects of the most important of these are described in terms of equilibrium positions, periodic motions, steady-state and transient motions. Also considered is the problem of attitude control of a spacecraft using active and/or passive methods of orientation and stabilization. In addition, a more advanced treatment of the development of attitude control systems is provided.

A description of the Earth's magnetic and gravitational fields allows us to clarify the relationship between natural features of the Earth's environment and the requirements of mission design, orbit construction and approaches to attitude control. A detailed Addendum provides an overview of circumstances on the Sun that render interplanetary space a very hazardous environment for spacecraft and for 'man in space'. The influence of this environment on spacecraft performance and survival is then presented, together with an outline of some of the mitigating strategies that can be invoked. A feature of the Addendum is the indication it provides of the challenges that the 
next generation of space experiments will pose to mission designers. It is accompanied by a separate set of references since if refers to ongoing work in space physics rather than to classical material.

A set of appendices contain mathematical tools useful in making derivations and in obtaining formulae, but without the inclusion of detailed numerical data. There are many suitable reference books that provide material in this regard, some of which are listed in the references to individual Chapters. Other references relevant to the Chapters are contained in footnotes.

While it is assumed that readers of this book are familiar with mathematical analysis, differential equation theory, analytical mechanics and the theory of stability, the intention of the authors in Chapters $1-13$ is to describe the main aspects of celestial mechanics and spaceflight dynamics without the use of complex mathematical formulae or the provision of examples of associated problem solving. This approach (breadth as opposed to depth) is for those readers who will deal in a general way only with space related topics. Chapters $14-$ 22 , which employ a more mathematically advanced treatment, are intended for readers interested at a specialist level in attitude dynamics and in the development of attitude control systems.

In the first fourteen chapters diagrams are frequently used to illustrate features of introductory spaceflight and attitude dynamics in lieu of complicated formula. In this regard, those innovative diagrams that were drawn manually by our co-author Boris Rauschenbakh, who died in 2001, are used in his memory with minimum changes. Only their inscriptions were rendered into English and, in some cases, an arrow over a letter or bold font was introduced to indicate a vector symbol. We hope that these visual presentations, with the historical dimension they provide through their association with Academician Rauschenbakh, will be attractive to those who are beginners in the area of astrodynamics, as well as to those who are already specialists, for whom they can serve to illuminate subtle details and provide new insights. The addition in footnotes of short historical biographies reflects the role of several centuries of international mathematicians in the achievement of space navigation and control.

The content concerned with spaceflight dynamics sprang initially from two courses of lectures for B.Sc., M.Sc. and Ph.D. students presented at the Moscow Institute of Physics and Technology. This 
material has been significantly expanded here as well as modulated by the practical experience of the authors in developing international space projects in engineering, control, mechanics, and space physics over a period extending from the early days of this discipline up to the present time.

It is a pleasure to thank our associates, colleagues and friends for their support during the preparation of this book, and for the many important general discussions and personal contacts with them that stimulated new ideas and contributed a depth of experience. Among those we would particularly like to acknowledge in this regard are D.Okhotsimsky, V.Sarychev, N.Trukhan, V.Pen'kov, V.Sazonov, V.Rauschenbakh, I.Ritous and V.Kozminikh, all of whom made greatly valued inputs. Also, we extend our thanks to V.Beletsky, F.Graziani, M.Hapgood and M.Dryer who read our manuscript and made several important remarks.

Michael Yu.Ovchinnikov,

Susan McKenna-Lawlor

Moscow, Russia

Maynooth, Ireland

ovchinni@keldysh.ru

stil@may.ie 


\section{About the Authors}

Rauschenbakh, Boris Victorovich (1915-2001). Doctor of Technical Science, Member of the Russian Academy of Sciences and the International Academy of Astronautics. One of the founders of practical cosmonautics in Russia. Worked in the 'Energia' Enterprise with Sergey Koroljev and later occupied the Chair of Theoretical Mechanics at the Moscow Institute of Physics and Technology.

Ovchinnikov, Michael Yurjevich, Doctor of Science in Physics and Mathematics at the Keldysh Institute of Applied Mathematics of the Russian Academy of Sciences and also a Professor at the Moscow Institute of Physics and Technology. Expert in mechanics, spaceflight dynamics and attitude control for small satellites.

McKenna-Lawlor, Susan, Emeritus Professor at the National University of Ireland, Maynooth. Guest Professor of the Chinese Academy of Sciences. Doctorate in Space Physics. Director of Space Technology Ireland, Ltd. Pioneering experiments flown on European, American, Russian and Chinese Space Agency Missions. Member of the International Academy of Astronautics. Recipient of the Tsiolkovsky Medal for Cosmonautics. 


\section{Table of Contents}

Preface $\quad$ v

About the Authors ix

1 Two-Body Problem 1

1.1 Equations of Motion ................ 1

1.2 Integral of Energy . . . . . . . . . . . . . . . 4

1.3 The Area Integral . . . . . . . . . . . . . . . 5

1.4 Laplace's Integral . . . . . . . . . . . . . . . . . 7

1.5 Kepler's Laws . . . . . . . . . . . . . . . . . . . . . . . 11

1.6 Kepler's Equation ................. 13

2 Qualitative Analysis of Orbits 17

2.1 Orbit Evolution . . . . . . . . . . . . . . . . . 17

2.2 Orbital Transfer . . . . . . . . . . . . . . . . . 18

2.3 SC Braking in the Atmosphere............. . 19

2.4 Interplanetary Flight ................ . 20

2.5 Circular Velocity .. . . . . . . . . . . . . . . 20

2.6 Escape Velocity . . . . . . . . . . . . . . . . . . 23

2.7 Hyperbolic Escape Velocity . . . . . . . . . . . . . 23

3 Perturbed Motion $\quad 25$

3.1 The $n$-Body Problem ............... 25

3.2 Planetocentric Form of Equations . . . . . . . . . 28

3.3 The Three-Body Problem ............. . 30

3.3.1 The Lagrange and Euler Cases . . . . . . . . . . 30

3.4 Restricted Three-Body Problem ............ 33

3.4.1 Hill's Surface .. . . . . . . . . . . . . . . 34

3.4.2 Singular Points of a Zero-Velocity Surface . . . 39 
4 Gravispheres 43

4.1 Sphere of Attraction . . . . . . . . . . . . . . 44

4.2 Sphere of Influence . . . . . . . . . . . . . . . . . 45

4.3 Kislik Gravisphere . . . . . . . . . . . . . . . 50

5 Osculating Elements $\quad 57$

5.1 Reference System and Orbital Elements . . . . . . . . . 57

5.2 Equations of Perturbed Motion . . . . . . . . . . 60

5.2.1 Introduction of Osculating Elements . . . . . . 61

5.2.2 Definition of the 'Main Operation' . . . . . . . 62

5.2.3 Equations for Osculating Elements . . . . . . 62

5.2.4 Transformation to the Argument of Latitude . . 69

6 Braking in the Earth's Atmosphere 71

6.1 Qualitative Analysis . . . . . . . . . . . 71

6.2 The Descending SC 'Paradox' . . . . . . . . . 76

7 Terrestrial Nonsphericity 79

7.1 Introduction to Models . . . . . . . . . . . . . . . . . . 79

7.2 Oblateness of the Gravitational Field . . . . . . . . 81

7.3 Calculation of Perturbing Accelerations . . . . . . . . 84

7.4 Evolution of the Equatorial Orbit . . . . . . . . . 86

7.5 Precession of the Inclined Orbit . . . . . . . . . . 88

7.6 Clarification of 'Inconsistency' . . . . . . . . . . . . . . 90

7.7 Orbits with Specific Inclinations . . . . . . . . . . 92

8 SC in the Field of Two Centers 97

9 Elements of Manoeuvring Theory 103

9.1 Statement of the Problem . . . . . . . . . . . . . 103

9.2 Orbital Plane Orientation Changing . . . . . . . . . . 108

9.3 In-plane Manoeuvres . . . . . . . . . . . . . . . . 111

9.3.1 Effect of a Tangent Pulse $\Delta V_{T} \ldots \ldots \ldots \ldots$

9.3.2 Effect of a Normal Pulse $\Delta V_{S} \ldots \ldots \ldots \ldots$. . . 114

9.3.3 Manoeuvre of Landing a SC . . . . . . . . . . 115

9.3.4 Change of the Period of SC Revolution . . . . . 117 
10 Trajectory Corrections 119

10.1 SC Motion Close to a Target Planet . . . . . . . . . 120

10.2 Segment of a Nominal Trajectory . . . . . . . . . 122

10.3 Properties of the Correction . . . . . . . . . . . 124

10.4 Two-parameter Correction . . . . . . . . . . . 126

10.5 Optimum Correction Point . . . . . . . . . . . 127

10.6 Singularity of the Correction Matrix . . . . . . . . 129

10.7 Correction Using the Singular Matrix . . . . . . . . 131

11 Rendezvous Manoeuvring 133

11.1 Control-Free Relative Motion . . . . . . . . . . . . 133

11.2 Approaches to Rendezvous Manoeuvring . . . . . . 135

12 Gravity-Assist Manoeuvre 139

12.1 Description of the Manoeuvre . . . . . . . . . . . 139

12.2 Application for Interplanetary Missions . . . . . . . . 143

12.3 Inclination Change of the Orbit . . . . . . . . . 145

13 About Orbit Determination 149

13.1 Least Square Method . . . . . . . . . . . . . 150

13.2 Concept of Recurrent Methods . . . . . . . . . . . 152

14 Introduction to Attitude Control 155

14.1 Types of Attitude Control Systems . . . . . . . . . 155

14.2 Scheme for SC Active Attitude Control . . . . . . . . 160

14.2.1 Estimation of Propellant Consumption . . . . . 162

14.2.2 Effect of a Constant Perturbing Torque . . . . . 165

14.3 SC Gyros for Attitude Control . . . . . . . . . . . . . . 169

14.3.1 Bounded Angular Momentum of a SC . . . . . 170

14.3.2 Unbounded Angular Momentum of a SC . . . . 171

15 Gravity-Gradient Torque Effect 173

15.1 General Assumptions . . . . . . . . . . . . . . . . 173

15.2 Reference Systems . . . . . . . . . . . . . . . 175

15.3 Kinematic Relationships . . . . . . . . . . . . 178

15.4 Gravity-Gradient Torque . . . . . . . . . . . . . . . . 179

15.5 Equations of SC Motion . . . . . . . . . . . 183 
16 SC Motion in a Circular Orbit $\quad 185$

16.1 Planar Motion of a SC . . . . . . . . . . . . 185

16.2 SC Equilibrium Positions . . . . . . . . . . . . . . 192

16.3 Sufficient Conditions for Stability . . . . . . . . 195

16.4 Necessary Conditions for Stability . . . . . . . . . . . . 197

17 SC Motion in an Elliptical Orbit 201

17.1 Equation of Planar SC Motion . . . . . . . . . . . 201

17.2 Linear Librations . . . . . . . . . . . . . . . . . . 203

17.2.1 Stability of the Solution of Mathieu's Equation 205

17.3 Non-linear Librations . . . . . . . . . . . . . . . . . 207

17.4 Periodic Motion of a SC . . . . . . . . . . . 209

17.4.1 Solutions for Slightly Elliptical Orbits . . . . . . 209

17.4.2 Numerical Investigation . . . . . . . . . . 212

18 Spinning Axisymmetric SC 219

18.1 Equations of Motion . . . . . . . . . . . . . . 219

18.2 Stationary Motions of a SC . . . . . . . . . . 221

18.3 Conditions of Stability . . . . . . . . . . . 224

19 Equilibrium of a Gyrostat 227

19.1 Equations of Motion . . . . . . . . . . . . . 227

19.2 Particular Cases of Equilibrium Positions . . . . . . . 229

20 Effect of Aerodynamic Torque 233

20.1 General Assumptions . . . . . . . . . . . . . . 233

20.2 Atmospheric Density Approximation . . . . . . . 237

20.3 Effect of the Earth's Rotation . . . . . . . . . . . 240

20.4 Equilibrium Positions . . . . . . . . . . . . . 242

21 SC in the Geomagnetic Field $\quad 247$

21.1 The Geomagnetic Field . . . . . . . . . . . . . . . 247

21.2 Models of the Geomagnetic Field . . . . . . . . 250

21.2.1 Dipole Terms $(n=1) \ldots \ldots . \ldots 250$

21.2.2 Quadrupole Terms $(n=2) \ldots \ldots . \ldots 253$

21.2.3 Octupole Terms $(n=3) \ldots \ldots . \ldots 254$

21.3 Equations of Motion . . . . . . . . . . . . 255

21.4 SC Planar Motion . . . . . . . . . . . . . . 259

21.4.1 Asymptotic Solution . . . . . . . . . 259 
21.4.2 Numerical Construction of Periodic Solutions . 260

21.4.3 Investigation of Stability . . . . . . . . 265

21.5 SC Spatial Motion . . . . . . . . . . . 268

22 Motion of a SC under Damping 275

22.1 Equations of Motion . . . . . . . . . . . 276

22.2 Fast Time. Equations of Motion in Standard Form . . 280

22.3 Averaging the Equations of Motion . . . . . . . . 283

22.4 Two Orthogonal Rods . . . . . . . . . . . . . . 285

22.4.1 Case without Hysteresis Rods . . . . . . . 286

22.4.2 Case of Weak Damping . . . . . . . . . . 287

Appendices

A Method of van der Pol 291

B Periodic Equations $\quad 293$

$\begin{array}{ll}\text { C Poincare's Method } & 297\end{array}$

$\begin{array}{ll}\text { Bibliography } & 299\end{array}$

Addendum. The Space Environment 303

A.1 Solar Activity and Near Earth Space . . . . . . . . . 303

A.1.1 The Sun and the Solar Wind . . . . . . . . . 303

A.1.2 Co-rotating Interaction Regions ... . . . . . 304

A.1.3 The Solar Cycle . . . . . . . . . . . . . . 305

A.1.4 Solar Flares . . . . . . . . . . . . . . . 307

A.1.5 Coronal Mass Ejections . . . . . . . . . . . . 307

A.1.6 Prediction of Proton Events . . . . . . . . . . 309

A.1.7 Numerical Modeling . . . . . . . . . . . . 310

A.1.8 The Earth's Magnetosphere ... . . . . . 312

A.1.9 Particle Populations in the Magnetosphere . . . 315

A.1.10 Galactic Cosmic Rays . . . . . . . . . . . 315

A.1.11 The Van Allen Belts . . . . . . . . . . . 316

A.1.12 Particle Motion in the Geomagnetic Field . . . 318

A.1.13 The South Atlantic Anomaly . . . . . . . . 320

A.1.14 Magnetic Storms . . . . . . . . . . . . . 320

A.1.15 External Magnetic Field Models . . . . . . . 323 
A.1.16 Relativistic Electrons . . . . . . . . . . 325

A.1.17 Thermospheric Heating . . . . . . . . . . . . 327

A.1.18 Atmospheric Drag . . . . . . . . . . . 327

A.1.19 Solar Radiation Pressure . . . . . . . . . . 330

A.1.20 Solar Sailing . . . . . . . . . . . . 332

A.1.21 Perturbing Effects on Orbiting SC . . . . . . . 332

A.1.22 Drag Free SC and their Applications . . . . . . 334

A.2 The Environment and SC Performance . . . . . . . 337

A.2.1 Electronics and Energetic Particle Radiation . . 337

A.2.2 Models of near Earth Energetic Particles . . . . 339

A.2.3 Radiation Models for Mission Evaluations . . . 340

A.2.4 SC Charging. . . . . . . . . . . . . . . . 342

A.2.5 SC Contamination . . . . . . . . . . . . 347

A.2.6 Sputtering from SC Surfaces . . . . . . . . 348

A.2.7 Corrosive Oxygen . . . . . . . . . . . . . . . . 348

A.2.8 Thermal Problems . . . . . . . . . . . . 350

A.2.9 Ambient Electric and Magnetic Fields . . . . 351

A.3 Overview of In-Orbit Disturbances . . . . . . . . 352

A.3.1 Surface and Internal Charging . . . . . . . . 356

A.3.2 Phantom Commands . . . . . . . . . . . . 358

A.3.3 Total Ionizing Dose and Single Event Effects . 359

A.3.4 Solar Cell Degradation and Displacement Damage 359

A.3.5 Loss of Attitude Control/Orientation . . . . . . 360

A.3.6 Loss of Signal Phase and Amplitude Lock . . 360

A.3.7 Solar Radio Interference . . . . . . . . . . . . . 361

A.3.8 Orbit Decay . . . . . . . . . . . . . 361

A.3.9 Biological Effects . . . . . . . . . . . . 362

A.3.10 Interplanetary Conditions . . . . . . . . . 364

A.4 Beyond the Solar System . . . . . . . . . . . . 365

List of References for Addendum $\quad 367$

Index of Scientists Cited in Footnotes 375

$\begin{array}{ll}\text { Index } & 377\end{array}$

$\begin{array}{ll}\text { List of Figures } & 391\end{array}$

$\begin{array}{ll}\text { List of Tables } & 397\end{array}$ 